\title{
RESEARCH
}

Open Access

\section{Knowledge, attitude, and preparedness toward IPV care provision among nurses and midwives in Tanzania}

\author{
Joel Seme Ambikile ${ }^{1,2}$, Sebalda Leshabari ${ }^{2}$ and Mayumi Ohnishi ${ }^{1,2^{*}}$ (D)
}

\begin{abstract}
Background: With increasing recognition of intimate partner violence (IPV) as a public health challenge, nurses and midwives are recognized for their crucial role in providing front-line healthcare services for IPV. This study aimed to evaluate knowledge, attitude, and preparedness related to IPV care provision in health facilities among nurses and midwives in Tanzania.

Methods: A self-administered anonymous questionnaire survey was conducted among nurses and midwives working in health facilities in the Mbeya region between December 2018 and January 2019. The questionnaire consisted of questions on their perceived and actual knowledge, attitudes, and preparedness to provide care in relation to IPV.
\end{abstract}

Results: A total of 662 (50.1\%) of 1321 nurses and midwives who worked in hospitals and/or health centers in the Mbeya region participated in this study, and 461 (69.6\%) completed questionnaires were included in the analysis. The proportion of nurses and midwives with high scores in IPV perceived knowledge, actual knowledge, attitude, and preparedness to provide care was 59.9\%, 53.1\%,54.2\%, and 54.0\%, respectively. Regardless of the type of facility, gender, educational level, and work experience, the use of IPV guidelines was significantly associated with high scores in IPV perceived knowledge $(P<0.001)$, actual knowledge $(P=0.004)$, and preparedness to provide care $(P<0.001)$, but not attitude, which was negatively associated $(P=0.048)$. Regardless of the type of facility, gender, educational level, and work experience, receiving preservice IPV training was associated with high scores in IPV perceived knowledge $(P<0.001)$, actual knowledge $(P=0.049)$, and preparedness to provide care $(P=0.002)$, but not attitude $(P=0.192)$. Regardless of the type of facility, gender, educational level, and work experience, inservice IPV training was associated with high scores in IPV perceived knowledge $(P<0.001)$, actual knowledge $(P=$ 0.043), and preparedness to provide care $(P=0.001)$, but not attitude $(P=0.063)$.

Conclusions: Although guidelines and training could improve nurses' and midwives' knowledge and preparedness to provide care regarding IPV, attitudes against IPV care are a challenge. To improve attitudes regarding IPV among front-line nurses and midwives, it is necessary to address concepts of IPV care and sympathy with potential and actual victims of IPV in pre- and in-service training in addition to providing recall-level knowledge.

Keywords: Intimate partner violence, Care provision, Health professionals

\footnotetext{
*Correspondence: mohnishi@nagasaki-u.ac.jp

'Department of Health Sciences, Nagasaki University Graduate School of Biomedical Sciences, 1-7-1 Sakamoto, Nagasaki-shi, Nagasaki-ken 8528520, Japan

${ }^{2}$ School of Nursing, Muhimbili University of Health and Allied Sciences, Dar es Salaam, Tanzania
}

(c) The Author(s). 2020 Open Access This article is licensed under a Creative Commons Attribution 4.0 International License, which permits use, sharing, adaptation, distribution and reproduction in any medium or format, as long as you give appropriate credit to the original author(s) and the source, provide a link to the Creative Commons licence, and indicate if changes were made. The images or other third party material in this article are included in the article's Creative Commons licence, unless indicated otherwise in a credit line to the material. If material is not included in the article's Creative Commons licence and your intended use is not permitted by statutory regulation or exceeds the permitted use, you will need to obtain permission directly from the copyright holder. To view a copy of this licence, visit http://creativecommons.org/licenses/by/4.0/ The Creative Commons Public Domain Dedication waiver (http://creativecommons.org/publicdomain/zero/1.0/) applies to the data made available in this article, unless otherwise stated in a credit line to the data. 


\section{Background}

Intimate partner violence (IPV) is defined as physical violence, sexual violence, stalking, and psychological aggression (including coercive acts) by a current or former intimate partner [1]. Worldwide, nearly one in three women is affected by IPV, with a higher 12-month prevalence in low-income than high-income countries, where the prevalence is less than 4\% [2]. With increased recognition of IPV as a public health problem, the World Health Organization (WHO) has presented effective prevention and intervention strategies for IPV [3]. This requires efforts that target specific risk and protective factors across individual, interpersonal, institutional, community, and societal levels. Such strategies include scaling up IPV services in statewide public health settings, including a patient-centered comprehensive response in clinical settings [4]. In this regard, the role of the health sector in identifying and supporting clients experiencing IPV cannot be overemphasized. This is because IPV clients appear in the healthcare system, but they are not screened and cared for by healthcare providers due to a lack of appropriate capacity; they are also not recognized as victims by healthcare providers [5].

Tanzania has reported a high 12-month IPV prevalence with a national rate of $44 \%$ [6]. IPV has serious maternal and neonatal consequences, with direct and indirect pathways that may lead to chronic health problems [7]. Recognition of the importance of addressing gender-based violence (GBV), including IPV, began in 2011 in collaboration with the US President's Emergency Plan for AIDS Relief. In the same year, the government developed the National Policy Guideline for the Health Sector Prevention and Response to Gender-Based Violence [8], which was instrumental to the development of the National Management Guidelines for the Health Sector Prevention and Response to GBV [9]. Although efforts are being made to integrate GBV care and services into existing service structures, the desired outcomes have not been sufficiently realized to satisfy IPV care and service needs [10]. Some of the reasons attributed to this situation are institutional barriers such as a lack of IPV knowledge, negative attitudes toward IPV, feeling unprepared, a lack of protocols and policies on IPV care provision, and time constraints at health facilities [11-13].

Because IPV is complex and multifaceted, a multisectoral approach is appropriate to address it [14]. One such approach is making IPV care and services available in health care facilities where IPV clients appear but are not recognized by healthcare providers [5]. Nurses and midwives constitute the largest health workforce in Tanzania [15] and are a core aspect of strengthening essential health services at the front line [16]. This puts them in a crucial position to address IPV from a facilitybased perspective. IPV knowledge, attitude, and preparedness are important factors that affect nurses' and midwives' capacity to manage clients experiencing IPV $[17,18]$ and are acquired through appropriate preservice and continuing education [19]. Therefore, evaluating nurses' and midwives' current conditions related to IPV knowledge, attitudes, and preparedness is important in determining their capacity to provide IPV care and services. This study was performed to evaluate the current conditions related to the knowledge, attitude, and preparedness of nurses and midwives to provide IPV care and services in Tanzania.

\section{Methods}

A cross-sectional study was conducted among nurses and midwives using an anonymous self-administered questionnaire in the Mbeya region, which has 7 districts and a population of three million, between December 2018 and January 2019. The region was selected due to a high lifetime IPV prevalence rate of $67 \%$, which is above the national average of $50.2 \%$ [6]. Forty health facilities including 15 hospitals and 25 health centers (HCs) in the Mbeya region were visited for data collection. A total of 1321 nurses and midwives were employed at health facilities at the time of data collection, and 662 (50.1\%) nurses who worked at hospitals and/or HCs during the day shift on the data collection day participated in the study. A total of 461 (69.6\%) completed questionnaires without missing data on the variables were included in this analysis. Different types of health facility establishments, such as government-, private-, and faith-based establishments, were included in the study. Respondents who were available and who consented at the time/day of data collection were recruited in this study. All shifts were considered for health facilities that had more than one working shift. Nurses and midwives who were not available at the time/day of the data collection due to being off duty, on holiday, or out for official duties and those who could not participate due to various reasons such as being sick and attending to serious patients were excluded from the data collection.

The Physician Readiness to Manage Intimate Partner Violence Survey (PREMIS) tool [20], a widely adopted, adapted, and used tool by various cadres in the health workforce [21-24], was applied to collect data after adjusting for nurses in the study setting. The tool was originally developed in English and translated into Swahili, the common language in Tanzania that most people are more comfortable with. Back translation was performed to ensure the accuracy and quality of the translation by researchers who spoke both languages. The Swahili questionnaire was then pretested among 12 nurses and midwives in two health facilities in different 
areas of the Mbeya region. Feedback from the pretest was used to clarify the questions, alter the sequence and organization of the questions, and adjust the allotted time for the questionnaire to improve it before data collection. The questionnaire consisted of questions with five parts: demographic characteristics; the Swahili version of PREMIS, including perceived and actual IPV knowledge as the cognitive domain; attitudes toward IPV and preparedness to provide IPV care and services as the affective domain; and the availability and use of guidelines for IPV services. Perceived knowledge of IPV was evaluated by 14 items, and responses were scored from 0 to 2. Actual knowledge of IPV was evaluated by 38 items; appropriate responses were scored as 1 and inappropriate responses as 0 . Overall knowledge scores were further calculated by summing the responses; the maximum scores were 28 and 38, respectively. Both knowledge scores were divided into two categories, high ( $\geq$ median) and low ( $<$ median). Attitude toward IPV was evaluated using 26 items rated on a 5-point Likert scale ranging from 0 (strongly disagree) to 4 (strongly agree). The overall IPV attitude score was calculated by summing the scores of each item; the maximum score was 104. The IPV attitude score was categorized as high $(\geq$ median) or low ( $<$ median). Preparedness to provide IPV care and services was assessed using 10 items rated on a 4-point Likert scale ranging from 0 (unprepared) to 3 (quite well prepared). The score for overall preparedness to provide IPV care was calculated by summing the scores of each item; the maximum score was 30 . The score for preparedness to provide IPV care and services was categorized as high ( $\geq$ median) or low ( $<$ median). All categories used were established with reference to a previous related study [25].

IBM SPSS version 20 was used to analyze the data. A chi-square test and logistic regression analysis were used to assess the association between demographic characteristics and IPV service-related knowledge, attitude, and preparedness among nurses. Spearman's rank correlation coefficient was used to measure the association between perceived IPV knowledge, actual IPV knowledge, IPV attitude, and preparedness to provide IPV services. In all analyses, $P<0.05$ was used to indicate statistical significance.

\section{Results}

A total of 662 nurses who worked in hospitals and/or HCs during the day shift on the day of data collection participated in the study, which constituted $50.1 \%$ of the 1321 nurses in total who were employed in the Mbeya region at the time of data collection. A total of 461 (69.6\%) fully completed questionnaires, with no missing data, were included in the analysis.
Table 1 shows the respondents' demographic information according to the health facility level.

The respective medians $(14,15,36$, and 14) for IPV perceived knowledge, actual knowledge, attitude, and preparedness to provide IPV care and services were used to categorize respondents with high and low scores. The proportion of respondents who had high IPV perceived knowledge, actual knowledge, attitude, and preparedness to provide IPV care and services was $59.9 \%, 53.1 \%, 54.2 \%$, and $54.0 \%$, respectively. The IPV survey tool used was tested for its internal consistency reliability, with a Cronbach's alpha of 0.758 for all four sets of items. The specific Cronbach's alphas for IPV perceived knowledge, actual knowledge, attitude, and preparedness to provide IPV care and services were $0.913,0.691,0.722$, and 0.945 , respectively. The proportion of use of IPV guidelines, such as standard operating procedures (SOPs) and protocols, among respondents was 272 (59.0\%).

Table 2 demonstrates the Spearman's rank correlation coefficient between the perceived and actual knowledge, attitude, and preparedness scores.

Table 3 shows the associations between demographic characteristics and perceived and actual knowledge, attitude, and preparedness of IPV care provision using the chi-square test.

Three models of logistic regression analyses were performed to analyze factors associated with perceived and actual knowledge, attitude, and preparedness for IPV care provision (Table 4). All models included the facility level and facility ownership as the work setting profile and gender, educational level, and work experience as the individual profile for the independent variables in addition to the use of guidelines for model $\mathrm{A}$, preservice training for model $\mathrm{B}$, and in-service training for model C.

\section{Discussion}

The findings of this study demonstrated that in general, nurses had insufficient knowledge, attitudes, and preparedness to provide competent IPV care and services. The level of the IPV cognitive domain, such as knowledge, did not translate into the same level of the affective domain, such as attitude, contrary to previous findings [25]. This study also highlights the role of IPV training and a supportive work environment in the provision of IPV care and services, such as the availability of guidelines regarding IPV care provision, consistent with previous studies conducted within and outside of Tanzania [10, 25-27].

The lack of IPV training experience in this study was consistent with previous studies in various settings [25, 28]. Although insufficient numbers of nurses had the opportunity to receive preservice training on IPV, nurses 
Table 1 Demographic characteristics according to the level of health facility $(N=461)$

\begin{tabular}{|c|c|c|c|c|c|}
\hline & \multicolumn{2}{|c|}{ Hospital $(n=384)$} & \multicolumn{2}{|c|}{ Health center $(n=77)$} & \multirow[t]{2}{*}{$P$ value } \\
\hline & $n$ & $\%$ & $n$ & $\%$ & \\
\hline \multicolumn{6}{|l|}{ Facility ownership } \\
\hline Government & 239 & 62.2 & 64 & 83.1 & \multirow[t]{2}{*}{$<0.001$} \\
\hline Non-government & 145 & 37.8 & 13 & 16.9 & \\
\hline \multicolumn{6}{|l|}{ Gender } \\
\hline Male & 113 & 29.4 & 22 & 28.6 & \multirow[t]{2}{*}{0.880} \\
\hline Female & 271 & 70.6 & 55 & 71.4 & \\
\hline \multicolumn{6}{|l|}{ Level of education } \\
\hline Certificate & 177 & 46.1 & 43 & 55.8 & \multirow[t]{2}{*}{0.118} \\
\hline Diploma/Bachelor's/Master's & 207 & 53.9 & 34 & 44.2 & \\
\hline \multicolumn{6}{|l|}{ Work experience } \\
\hline$\leq 5$ years & 116 & 43.2 & 29 & 37.7 & \multirow[t]{2}{*}{0.367} \\
\hline$>5$ years & 218 & 56.8 & 48 & 62.3 & \\
\hline \multicolumn{6}{|l|}{ Use of guidelines" } \\
\hline No & 156 & 40.6 & 33 & 42.9 & \multirow[t]{2}{*}{0.716} \\
\hline Yes & 228 & 59.4 & 44 & 57.1 & \\
\hline \multicolumn{6}{|l|}{ Preservice IPV training } \\
\hline No/do not remember & 266 & 69.3 & 63 & 81.8 & \multirow[t]{2}{*}{0.026} \\
\hline Yes & 118 & 30.7 & 14 & 18.2 & \\
\hline \multicolumn{6}{|l|}{ In-service IPV training } \\
\hline No/do not remember & 294 & 76.6 & 65 & 84.4 & \multirow[t]{2}{*}{0.130} \\
\hline Yes & 90 & 23.4 & 12 & 15.6 & \\
\hline
\end{tabular}

A chi-square test was conducted

\#Availability and use of standard operating procedure (SOP) or protocol/guideline for IPV services

with shorter work experience, who might be younger and may have recently received nursing education including midwifery education, were more likely to report having preservice training on IPV regardless of educational level. In Tanzania, IPV care provision could be introduced into in-service nursing training even if topics related to IPV are not fully covered during preservice training. Nurses with longer work experience reported being more likely to have had opportunities for inservice training on IPV. Furthermore, nurses with preservice training on IPV were more likely to have had opportunities for in-service training on IPV. There was no association in this study between work experience and the knowledge, attitude, and preparedness scores for IPV care provision. The findings of this study support the role of preservice and in-service IPV training in improving nurses' capacity to provide IPV services, as corroborated by previous studies $[20,21]$. The recently developed WHO IPV curriculum for training healthcare providers [29] needs to be integrated into existing preservice nursing curricula in Tanzania, and the content should include basic/background knowledge on IPV, IPV screening and assessment, offering front-line support to clients who disclose IPV, IPV documentation, IPV referral sources, and orientation to IPV guidelines as per the $\mathrm{WHO}$ recommendations [30]. Training on

Table 2 Correlations between IPV perceived knowledge, actual knowledge, attitude, and preparedness scores $(N=461)$

\begin{tabular}{|c|c|c|c|c|c|c|}
\hline & \multicolumn{2}{|c|}{ IPV perceived knowledge } & \multicolumn{2}{|c|}{ IPV actual knowledge } & \multicolumn{2}{|c|}{ IPV attitude } \\
\hline & $\rho$ & $P$ value & $\rho$ & $P$ value & $\rho$ & $P$ value \\
\hline Perceived IPV knowledge & 1 & & & & & \\
\hline Actual IPV knowledge & 0.171 & $<0.001$ & 1 & & & \\
\hline IPV attitude & -0.189 & $<0.001$ & -0.163 & $<0.001$ & 1 & \\
\hline Preparedness to provide IPV care & 0.408 & $<0.001$ & 0.172 & $<0.001$ & -0.143 & 0.002 \\
\hline
\end{tabular}

Spearman's rank correlation coefficient $(\rho)$ was calculated 
Table 3 Associations between demographic characteristics and IPV perceived knowledge, actual knowledge, attitude, and preparedness scores $(N=461)$

\begin{tabular}{|c|c|c|c|c|c|c|c|c|c|c|c|c|}
\hline & \multicolumn{2}{|c|}{$\begin{array}{l}\text { IPV perceived } \\
\text { knowledge } \\
\text { (high } \geq 14 \text { ) }\end{array}$} & \multirow[t]{2}{*}{$P$ value } & \multicolumn{2}{|c|}{$\begin{array}{l}\text { IPV actual } \\
\text { knowledge } \\
\text { (high } \geq 15 \text { ) }\end{array}$} & \multirow[t]{2}{*}{$\begin{array}{l}P \\
\text { value }\end{array}$} & \multicolumn{2}{|c|}{$\begin{array}{l}\text { IPV attitude } \\
\text { (high } \geq 36 \text { ) }\end{array}$} & \multirow[t]{2}{*}{$\begin{array}{l}P \\
\text { value }\end{array}$} & \multicolumn{2}{|c|}{$\begin{array}{l}\text { IPV care } \\
\text { preparedness } \\
\text { (high } \geq 14 \text { ) }\end{array}$} & \multirow[t]{2}{*}{$P$ value } \\
\hline & $\bar{n}$ & $\%$ & & $\bar{n}$ & $\%$ & & $\bar{n}$ & $\%$ & & $\bar{n}$ & $\%$ & \\
\hline \multicolumn{13}{|l|}{ Level of facility } \\
\hline Hospital & 233 & 60.7 & 0.430 & 193 & 50.3 & 0.006 & 205 & 53.4 & 0.416 & 205 & 53.4 & 0.546 \\
\hline Health center & 43 & 55.8 & & 52 & 67.5 & & 45 & 58.4 & & 44 & 57.1 & \\
\hline \multicolumn{13}{|l|}{ Facility ownership } \\
\hline Government & 172 & 56.8 & 0.060 & 159 & 52.5 & 0.690 & 170 & 56.1 & 0.263 & 157 & 51.8 & 0.190 \\
\hline Non-government & 104 & 65.8 & & 86 & 54.4 & & 80 & 50.6 & & 92 & 58.2 & \\
\hline \multicolumn{13}{|l|}{ Gender } \\
\hline Male & 91 & 67.4 & 0.034 & 80 & 59.3 & 0.090 & 71 & 52.6 & 0.650 & 82 & 60.7 & 0.062 \\
\hline Female & 185 & 56.7 & & 165 & 50.6 & & 179 & 54.9 & & 167 & 52.1 & \\
\hline \multicolumn{13}{|l|}{ Level of education } \\
\hline Certificate & 124 & 56.4 & 0.142 & 116 & 52.7 & 0.864 & 121 & 55.0 & 0.751 & 117 & 53.2 & 0.732 \\
\hline Diploma/Bachelor's/Master's & 152 & 63.1 & & 129 & 53.5 & & 129 & 53.5 & & 132 & 54.8 & \\
\hline \multicolumn{13}{|l|}{ Work experience } \\
\hline$\leq 5$ years & 127 & 65.1 & 0.049 & 96 & 49.2 & 0.149 & 108 & 55.4 & 0.670 & 108 & 55.4 & 0.613 \\
\hline$>5$ years & 149 & 56.0 & & 149 & 56.0 & & 142 & 53.4 & & 141 & 53.0 & \\
\hline \multicolumn{13}{|l|}{ Use of guidelines ${ }^{\#}$} \\
\hline No & 91 & 48.1 & $<0.001$ & 86 & 45.5 & 0.006 & 112 & 59.3 & 0.071 & 82 & 43.4 & $<0.001$ \\
\hline Yes & 185 & 68.0 & & 159 & 58.5 & & 138 & 50.7 & & 167 & 61.4 & \\
\hline \multicolumn{13}{|l|}{ Preservice IPV training } \\
\hline No/does not remember & 163 & 59.1 & $<0.001$ & 167 & 68.2 & 0.105 & 185 & 56.2 & 0.173 & 162 & 49.2 & 0.001 \\
\hline Yes & 113 & 40.9 & & 78 & 31.8 & & 65 & 49.2 & & 87 & 65.9 & \\
\hline \multicolumn{13}{|l|}{ In-service IPV training } \\
\hline No/does not remember & 194 & 54.0 & $<0.001$ & 181 & 50.4 & 0.028 & 204 & 56.8 & 0.036 & 179 & 49.9 & 0.001 \\
\hline Yes & 82 & 80.4 & & 64 & 62.7 & & 46 & 45.1 & & 70 & 68.6 & \\
\hline
\end{tabular}

A chi-square test was conducted

${ }^{\#}$ Availability and use of standard operating procedure (SOP) or protocol/guideline for IPV services

Table 4 Associations between demographic characteristics and IPV-related perceived and actual knowledge, attitude, and preparedness $(N=461)$

\begin{tabular}{|c|c|c|c|c|c|c|c|c|c|c|}
\hline \multicolumn{3}{|c|}{$\begin{array}{l}\text { IPV perceived } \\
\text { knowledge score } \\
\text { (high } \geq 14 \text { ) }\end{array}$} & \multicolumn{3}{|c|}{$\begin{array}{l}\text { IPV actual } \\
\text { knowledge score } \\
\text { (high } \geq 15 \text { ) }\end{array}$} & \multicolumn{3}{|c|}{$\begin{array}{l}\text { IPV attitude } \\
\text { score (high } \geq 36 \text { ) }\end{array}$} & \multicolumn{2}{|c|}{$\begin{array}{l}\text { IPV care } \\
\text { preparedness score } \\
\text { (high } \geq 14 \text { ) }\end{array}$} \\
\hline $\mathrm{AOR}$ & $95 \% \mathrm{Cl}$ & $P$ & $\overline{A O R}$ & $95 \% \mathrm{Cl}$ & $P$ & $\mathrm{AOR}$ & $95 \% \mathrm{Cl}$ & $P$ & $\overline{A O R}$ & $95 \%$ Cl \\
\hline
\end{tabular}

\begin{tabular}{|c|c|c|c|c|c|c|c|c|c|c|c|c|}
\hline \multicolumn{13}{|l|}{ Model A } \\
\hline Use of guideline (ref: no) & 2.700 & $\begin{array}{l}1.802, \\
4.044\end{array}$ & $\begin{array}{l}< \\
0.001\end{array}$ & 1.786 & $\begin{array}{l}1.209 \\
2.639\end{array}$ & 0.004 & 0.678 & $\begin{array}{l}0.462 \\
0.996\end{array}$ & 0.048 & 2.266 & $\begin{array}{l}1.533, \\
3.350\end{array}$ & $\begin{array}{l}< \\
0.001\end{array}$ \\
\hline \multicolumn{13}{|l|}{ Model B } \\
\hline $\begin{array}{l}\text { Preservice IPV training (ref: no/does not } \\
\text { remember) }\end{array}$ & 5.793 & $\begin{array}{l}3.368 \\
9.967\end{array}$ & $\begin{array}{l}< \\
0.001\end{array}$ & 1.536 & $\begin{array}{l}1.001 \\
2.355\end{array}$ & 0.049 & 0.757 & $\begin{array}{l}0.499 \\
1.150\end{array}$ & 0.192 & 1.972 & $\begin{array}{l}1.279 \\
3.039\end{array}$ & 0.002 \\
\hline \multicolumn{13}{|l|}{ Model C } \\
\hline $\begin{array}{l}\text { In-service IPV training (ref: no/does not } \\
\text { remember) }\end{array}$ & 3.638 & $\begin{array}{l}2.110 \\
6.274\end{array}$ & $\begin{array}{l}< \\
0.001\end{array}$ & 1.618 & $\begin{array}{l}1.016 \\
2.676\end{array}$ & 0.043 & 0.652 & $\begin{array}{l}0.415 \\
1.023\end{array}$ & 0.063 & 2.209 & $\begin{array}{l}1.371 \\
3.560\end{array}$ & 0.001 \\
\hline
\end{tabular}

Logistic regression analysis was conducted. Level of facility, facility ownership, gender, level of professional education, and work experience were adjusted for in each model

$\mathrm{Cl}$ confidence interval

\#Availability and use of standard operating procedures (SOPs) or protocols/guidelines for IPV services 
IPV care provision is one of the essential factors to improve nurses' care capacity; therefore, it is important to integrate IPV care provision into preservice training across the different levels of nursing education and to ensure continuous in-service training, which guarantees and maintains their IPV care capacity and up-to-date knowledge and skills. At the same time, nurses without preservice training on IPV care must become capable of IPV care provision through in-service training because they are front-line health professionals for IPV care. Moreover, this study emphasizes the role of IPV guidelines in supporting the provision of IPV care and services as emphasized by the WHO [31]. In this study, nurses with IPV training experience were more likely to use guidelines. The availability of guidelines and protocols for care provision at health facilities is fundamental, as is the introduction of and training to appropriately utilize guidelines and protocols.

Appropriate and effective IPV educational methods, such as interactive learning and practical applications of learning, may be used [32] after they are adapted to the local context. In this study, there were no associations between educational level, work experience, training opportunities, and the affective domain, such as attitudes toward IPV care. Strengthening the affective domain requires active learning and interactive education, such as case studies, group discussions, role play, and case presentations for affective IPV learning [33], to stimulate the ability to apply, analyze, evaluate, and create interpretations and problem solving.

There are several limitations to this study. First, we did not consider whether the respondents were representative of nurses in Tanzania, although we attempted to recruit respondents from different types of health facilities including hospitals and HCs throughout the region. The demographic characteristics of the study participants from hospitals and HCs likely do not differ from those in other regions in Tanzania. Thus, the findings from this study may demonstrate the actual conditions of IPV-related care capacities in regional areas in Tanzania. Second, only respondents without missing data were included in the final analysis. The excluded respondents may have differed with regard to background information such as educational status, work experience, and IPV training experience. Because of these limitations, the samples we analyzed in this study might be biased toward nurses with higher educational levels. However, there have been limitations in IPV-related care at higher-level health care facilities such as hospitals, which employ nursing personnel with higher educational levels. Thus, conditions of health care provision at dispensaries, which are the lowest level of the health care system in Tanzania and are not addressed in this study, should be extremely limited, including the capacity of nursing personnel and the support system for them. Third, this study was based on self-reported data that may be subjective because we did not observe actual IPV service provision. Therefore, the psychomotor domain was not evaluated in this study, and a comprehensive capacity for IPV care and service provision was not determined because the integration of the cognitive, affective, and psychomotor domains was not analyzed. Future studies should examine the effects of the affective and psychomotor domains on IPV-related care provisions, which would contribute to improving nursing personnel capacity building. Finally, the scores for the internal consistency reliability of the scales used for evaluating actual IPV knowledge and IPV attitude were not sufficiently high. Evaluating actual capacity and performance in providing IPV care requires the use of tools and methodologies to assess the affective and psychomotor domains, including performance tests.

\section{Conclusions}

This study reported actual conditions of knowledge, attitude, and preparedness in relation to IPV care provision among nurses in Tanzania, despite several limitations. The respondents' attitudes toward IPV care present a challenge for enhancement, although guidelines and training could improve nurses' knowledge and preparedness to provide care regarding IPV. To strengthen appropriate attitudes toward IPV among front-line nurses, it is necessary to address their thoughts about IPV care and sympathy with potential and actual victims of IPV in pre- and in-service training rather than providing only recall-level knowledge.

\section{Abbreviations}

AIDS: Acquired immunodeficiency syndrome; AOR: Adjusted odds ratio; Cl: Confidence interval; HIV: Human immunodeficiency virus; SOPs: Standard operating procedures

\section{Acknowledgements}

We wish to express our appreciation to the nurses and midwives who participated in this study. We are also grateful to the health authorities of the Mbeya districts and the leaders of the hospitals, HCs, and dispensaries for their support and collaboration in this study.

\section{Authors' contributions \\ JSA conceived the study and its design, collected the data, performed the data analysis, and drafted the manuscript. MO and SL reviewed the design, participated in the data collection, and supervised the data analysis and manuscript preparation. All authors contributed equally to the research and writing of the manuscript and read and approved the final manuscript.}

\section{Funding}

This study was sponsored by the Japan Society for the Promotion of Science through the RONPAKU program. The author's views expressed in this manuscript do not necessarily reflect the views of the Japan Society for the Promotion of Science.

Availability of data and materials

The datasets used and/or analyzed during the current study are available from the corresponding author on reasonable request. 


\section{Ethics approval and consent to participate}

This study was approved by the Ethics Committees of Nagasaki University in Japan (approval number: 18061432), Muhimbili University of Health and Allied Sciences in Tanzania, and the National Institute of Medical Research in Tanzania (approval number NIMR/HQ/R.8a/Nol. IX/2948). Mbeya Zonal Referral Hospital approved the research proposal and agreed to allow the study to be conducted at the institution. Permission to conduct the study was granted by the Regional Medical Officer, district medical officers, and all health facilities that were involved. Confidentiality was guaranteed by ensuring the participants' anonymity and obtaining written informed consent from all participants. Participation was voluntary, and withdrawal from the study was an option for respondents at any time.

\section{Consent for publication}

The study participants completed the questionnaire and submitted the completed questionnaire in the designated place after receiving verbal and written ethical explanations of the study's purposes, methods, anonymous data collection and analysis processes, confidentiality, and publication. This research article does not include any individual participant data, such as images, videos, or voice recordings.

\section{Competing interests}

The authors declare that they have no competing interests.

\section{Received: 29 November 2019 Accepted: 23 July 2020}

\section{Published online: 03 August 2020}

\section{References}

1. Breiding MJ, Basile KC, Smith SG, Black MC, Mahendra R. Intimate partner violence surveillance uniform definitions and recommended data elements: National Center for Injury Prevention and Control. 2015. https://www.cdc. gov/violenceprevention/pdf/ipv/intimatepartnerviolence.pdf. Accessed 15 Nov 2019

2. Heise $L L$, Kotsadam A. Cross-national and multilevel correlates of partner violence: an analysis of data from population-based surveys. Lancet Glob Health. 2015:3:e332-40.

3. World Health Organization/London School of Hygiene and Tropical Medicine. Preventing intimate partner and sexual violence against women: taking action and generating evidence. Geneva: World Health Organization/ London School of Hygiene and Tropical Medicine; 2010.

4. Dutton MA, James L, Langhorne A, Kelley M. Coordinated public health initiatives to address violence against women and adolescents. J Womens Health (Larchmt). 2015:24:80-5.

5. Rodríguez M, Valentine JM, Son JB, Muhammad M. Intimate partner violence and barriers to mental health care for ethnically diverse populations of women. Trauma Violence Abuse. 2009;10:358-74.

6. National Bureau of Statistics Tanzania and ICF Macro. Tanzania demographic and health survey 2010. Dar es Salaam, Tanzania: National Bureau of Statistics Tanzania and ICF Macro; 2011.

7. Howard LM, Oram S, Galley H, Trevillion K, Feder G. Domestic violence and perinatal mental disorders: a systematic review and meta-analysis. PLoS Med. 2013;10:e1001452.

8. Ministry of Health and Social Sevices. Summary of national policy guideline for the health sector prevention and response to gender-based violence (GBV). Dar es Salaam, Tanzania: Ministry of Health and Social Services; 2011.

9. Ministry of Health and Social Welfare. National management guidelines for the health sector response to and prevention of gender-based violence (GBV). Dar es Salaam, Tanzania: Ministry of Health and Social Welfare; 2011.

10. McCleary-Sills J, Namy S, Nyoni J, Rweyemamu D, Steven E, Salvatory A. Help-seeking pathways and barriers for survivors of gender-based violence in Tanzania: results from a study in Dar es Salaam, Mbeya, and Iringa Regions. Dar es Salaam, Tanzania: EngenderHealth/CHAMPION; 2013.

11. Guruge S. Nurses' role in caring for women experiencing intimate partner violence in the Sri Lankan context. ISRN Nurs. 2012;2012:486273.

12. Sundborg EM, Saleh-Stattin N, Wändell P, Törnkvist L. Nurses' preparedness to care for women exposed to intimate partner violence: a quantitative study in primary health care. BMC Nurs. 2012;11:1.

13. Hamberger LK, Rhodes K, Brown J. Screening and intervention for intimate partner violence in healthcare settings: creating sustainable system-level programs. J Womens Health (Larchmt). 2015;24:86-91.
14. Ali P. Gender-based violence and the role of healthcare professionals. Nurs Open. 2018:5:4-5.

15. Exavery A, Lutambi AM, Wilson N, Mubyazi GM, Pemba S, Mbaruku G. Gender-based distributional skewness of the United Republic of Tanzania's health workforce cadres: a cross-sectional health facility survey. Hum Resour Health. 2013;11:28.

16. World Health Organization. Global strategic directions for strengthening nursing and midwifery 2016-2020. 2016. https://www.who.int/hrh/nursing_ midwifery/global-strategic-midwifery2016-2020.pdf?ua=1. Accessed 15 Nov 2019.

17. Ramsay J, Rutterford C, Gregory A, Dunne D, Eldridge S, Sharp D, et al. Domestic violence: knowledge, attitudes, and clinical practice of selected UK primary healthcare clinicians. Br J Gen Pract. 2012;62:e647-55.

18. Gomez-Fernandez MA, Goberna-Tricas J, Paya-Sanchez M. Intimate partner violence as a subject of study during the training of nurses and midwives in Catalonia (Spain): a qualitative study. Nurse Educ Pract. 2017;27:13-21.

19. Sharma KK, Vatsa M, Kalaivani M, Bhardwaj DN. Knowledge, attitude, practice and learning needs of nursing personnel related to domestic violence against women: a facility based cross sectional survey. Int J Community Med Public Health. 2018;5:996-1003.

20. Short LM, Alpert E, Harris JM Jr, Surprenant ZJ. A tool for measuring physician readiness to manage intimate partner violence. Am J Prev Med. 2006;30:173-80.

21. Papadakaki M, Petridou E, Kogevinas M, Lionis C. Measuring the effectiveness of an intensive IPV training program offered to Greek general practitioners and residents of general practice. BMC Med Educ. 2013;13:46.

22. Goicolea I, Hurtig AK, San Sebastian M, Vives-Cases C, Marchal B. Developing a programme theory to explain how primary health care teams learn to respond to intimate partner violence: a realist case-study. BMC Health Serv Res. 2015;15:228.

23. Goicolea I, Marchal B, Hurtig AK, Vives-Cases C, Briones-Vozmediano E, San SM. Why do certain primary health care teams respond better to intimate partner violence than others? A multiple case study. Gac Sanit. 2019:33:169_ 76

24. Barnard M, West-Strum D, Yang Y, Holmes E. Evaluation of a tool to measure pharmacists' readiness to manage intimate partner violence. Pharmacy (Basel). 2018:6:66.

25. Mengistu D, Zeleke $H$, Alem G. Assessment of nurses? Preparedness and identify barriers to care women exposed to intimate partner violence in East Gojjam Zone, Ethiopia, 2014. J Nurs Care. 2015:4:2-10.

26. Guillery ME, Benzies KM, Mannion C, Evans S. Postpartum nurses' perceptions of barriers to screening for intimate partner violence: a crosssectional survey. BMC Nurs. 2012;11:2

27. Sprague S, Swaminathan A, Slobogean GP, Spurr H, Arseneau E, Raveendran $L$, et al. A scoping review of intimate partner violence educational programs for health care professionals. Women Health. 2018:58:1192-206.

28. Crombie N, Hooker L, Reisenhofer S. Nurse and midwifery education and intimate partner violence: a scoping review. J Clin Nurs. 2017;26:2100-25.

29. World Health Organization. Caring for women subjected to violence: a curriculum for training health-care providers [Internet]. 2019. 94 p. Available from: https://apps.who.int/iris/bitstream/handle/10665/330084/97892415171 02-eng.pdf.

30. World Health Organization. Responding to intimate partner violence and sexual violence against women. 2013. https://apps.who.int/iris/bitstream/ handle/10665/85240/9789241548595_eng.pdf?sequence=1. Accessed 15 Nov 2019

31. World Health Organization. Strengthening health systems to respond to women subjected to intimate partner violence or sexual violence: a manual for health managers. 2017. https://apps.who.int/iris/bitstream/handle/1 0665/259489/9789241513005-eng.pdf?sequence=1. Accessed 11 Nov 2019.

32. Sammut D, Kuruppu J, Hegarty K, Bradbury-Jones C. Which violence against women educational strategies are effective for prequalifying health-care students?: a systematic review. Trauma Violence Abuse. 2019. https://doi. org/10.1177/1524838019843198.

33. Ondrejka D. Affective teaching in nursing. New York: Springer Publishing Company; 2014

\section{Publisher's Note}

Springer Nature remains neutral with regard to jurisdictional claims in published maps and institutional affiliations. 